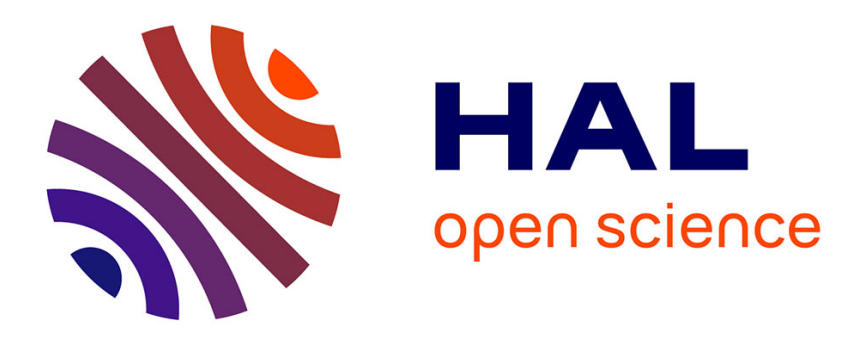

\title{
Générateur d'impulsions pour expérience de R. M. N. à impulsions multiples
}

\author{
Daniel Bloyet, S.K. Kan
}

\section{To cite this version:}

Daniel Bloyet, S.K. Kan. Générateur d'impulsions pour expérience de R. M. N. à impulsions multiples. Revue de Physique Appliquée, 1971, 6 (4), pp.475-480. 10.1051/rphysap:0197100604047500 . jpa00243573

\section{HAL Id: jpa-00243573 https://hal.science/jpa-00243573}

Submitted on 1 Jan 1971

HAL is a multi-disciplinary open access archive for the deposit and dissemination of scientific research documents, whether they are published or not. The documents may come from teaching and research institutions in France or abroad, or from public or private research centers.
L'archive ouverte pluridisciplinaire HAL, est destinée au dépôt et à la diffusion de documents scientifiques de niveau recherche, publiés ou non, émanant des établissements d'enseignement et de recherche français ou étrangers, des laboratoires publics ou privés. 


\title{
GÉNÉRATEUR D'IMPULSIONS POUR EXPÉRIENCE DE R. M. N. A IMPULSIONS MULTIPLES
}

\author{
D. BLOYET et S. K. KAN \\ Institut d'Electronique Fondamentale $(*)$, \\ Université Paris, XIe, Centre d'Orsay, 91-Orsay
}

(Reçu le 11 août 1971)

\begin{abstract}
Résumé. - Nous décrivons ci-dessous un générateur d'impulsions à technique digitale spécialement adapté aux expériences de Résonance Magnétique Nucléaire pulsée. Ce générateur délivre en mode déclenché ou relaxé une séquence de 8 impulsions et de 8 écarts de durées réglables indépendamment les unes des autres.

Nous donnons un certain nombre de détails sur son principe et sur le fonctionnement des éléments logiques fondamentaux : compteurs décimaux, comparateurs de nombres digitaux, commutateurs de données, problèmes de déclenchement et de synchronisation, technique de décodages, etc.

Ce type d'appareil allie à une grande simplicité d'emploi la précision sur chaque durée préaffichée.

Abstract. - We describe here a programmable digital pulse generator primarily intended for use in pulsed nuclear magnetic resonance (N. M R.) experiments. It generates in the freerun or triggered mode eight separately adjustable impulses and intervals of 0.1 to $99,9 \mu \mathrm{s}$ in steps of $0.1 \mu \mathrm{s}$ and 1 to $999 \mu$ s in steps of $1 \mu$ s respectively when a $10 \mathrm{MHz}$ clock frequency is used.

Details are given concerning its principle, choice of logical circuits, method for jitter suppression when used in the triggered mode, synchronisation, series to parallel output conversion technique, etc.

Its main advantages are : simplicity in circuitry and utilisation, programmable, high resolution as well as stability.
\end{abstract}

1. Introduction. - La réponse d'un système de spins à un champ radiofréquence est habituellement étudiée dans deux cas limites. Dans l'un le champ radiofréquence est inférieur au champ de saturation et le spectre de raies est balayé très lentement soit en champ soit en fréquence. Dans l'autre un champ radiofréquence de très forte intensité est appliqué pendant un temps très court devant $T_{2}$ et le signal de précession libre est observé. Dans les deux cas les informations extraites du signal sont identiques et seules des raisons pratiques déterminent le choix de l'une ou de l'autre méthode.

Il n'en est pas de même lorsqu'on applique une séquence d'impulsions de longueur variable car des informations supplémentaires peuvent être obtenues. C'est notamment le cas de deux impulsions $90^{\circ}$ (Hahn [1]) ou 90-180 (Carr-Purcell) [2]. Lorsque plus de deux impulsions sont mises en jeu un paramètre supplémentaire intéressant peut être même introduit c'est la phase relative du champ radiofréquence au début de chaque impulsion. On dispose ainsi d'un moyen pour faire varier la direction de $H_{1}$ dans le plan équatorial du trièdre tournant.

(*) Bâtiment 220, Laboratoire associé au C. N. R. S.
Il est ainsi possible d'annuler l'effet de l'interaction dipolaire et quadrupolaire dans les solides ou de diminuer à volonté l'influence des déplacements chimiques en R. M. N. haute résolution [3].

Le générateur d'impulsions qui est décrit a été conçu pour réaliser ces expériences, il permet à partir d'une horloge pilote à quartz de créer huit impulsions de largeur réglable de $0,1 \mu$ s à $99,9 \mu$ s avec un incrément de $0,1 \mu \mathrm{s}$ et huit écarts réglables de $1 \mu \mathrm{s}$ à $999 \mu \mathrm{s}$ avec un incrément de $1 \mu \mathrm{s}$. L'appareil est très versatile par rapport aux appareils déjà décrits [4] et peut indifféremment être utilisé comme générateur d'impulsions en mode série ou parallèle.

2. Principe. - L'horloge à quartz qui pilote le générateur radiofréquence est utilisée comme base de temps pour le fonctionnement du générateur d'impulsions. Chaque durée d'impulsion ou d'écart est définie par un certain nombre de périodes de l'horloge pilote comptées par un compteur électronique. Chacune est affichée par un des 16 groupes de commutateurs qui précisent le nombre de périodes d'horloge correspondantes.

Un comparateur de chiffres binaires code BCD 1,2, 4, 8 permet de comparer l'état du compteur de périodes et l'état du commutateur d'affichage. Lorsque les 
deux états sont identiques, c'est-à-dire lorsque le compteur a totalisé le nombre de périodes affichées, un dispositif de commutation permet de passer au comptage des périodes qui définiront la durée de l'impulsion ou de l'écart suivant.

Pour permettre une plus grande souplesse d'emploi les durées d'impulsions et les écarts entre impulsions peuvent être déterminés soit à partir de deux horloges dont les périodes $\theta_{\mathrm{i}}$ et $\theta_{\mathrm{e}}$ sont dans un rapport variable, soit à partir de deux horloges indépendantes.

Le schéma de principe de l'appareil est représenté sur la figure 1 .

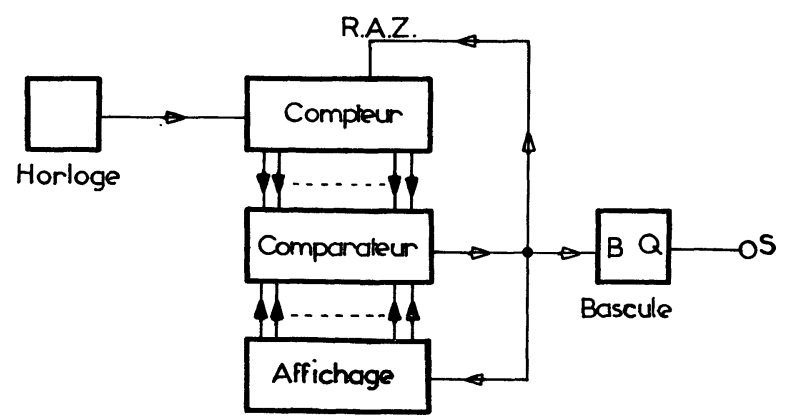

Fig. 1. - Schéma de principe du générateur d'impulsions.

A l'instant $t=0$, le compteur se trouve en position « 0 », la bascule B en position $Q=0$ et le commutateur d'affichage indique, en nombre $N_{1}$ de périodes d'horloge $\theta_{i}$, la durée de la première impulsion. Dès que l'horloge est reliée au compteur ce dernier totalise le nombre $N$ de périodes reçues. Tant que $N<N_{1}$ le comparateur délivre un niveau « 0 ", par contre dès que $N$ atteint la valeur $N_{1}$ le comparateur délivre un niveau « 1 » qui commande les quatre opérations suivantes :

a) remise du compteur à zéro,

b) basculement de la bascule bistable $\mathrm{B}$ en position $Q=1$,

c) commutation du comparateur pour qu'il voie le nombre $N_{2}$ correspondant à la durée de l'écart suivant la première impulsion,

d) commutation éventuelle des deux horloges.

Dès cet instant le comptage recommence pour l'écart de durée $N_{2}$ en périodes d'horloge $\theta_{\mathrm{e}}$.

La bascule B reste en position $Q=0$ pendant le temps $T_{1}=N_{1} \theta_{\mathrm{i}}$ qui fixe la durée de la première impulsion, en position $Q=1$ pendant $T_{2}=N_{2} \theta_{\mathrm{e}}$ qui fixe la durée du premier écart et ainsi de suite.

Les deux principaux problèmes posés pour la réalisation de ce dispositif sont la réalisation $\mathrm{du}$ comparateur et sa commutation à chaque fin de cycle de comptage vers le commutateur d'affichage suivant.

3. Description des circuits. - 3.1 Le COMPARATEUR. - a) Principe. - L'élément de base de comparaison de deux nombres digitaux est le circuit « ou exclusif $»$.
La fonction « ou exclusif » (symbole $\oplus$ ) est donnée par l'équation suivante : $A \oplus B=A \bar{B}+B \bar{A}$, représentée par la table de vérité de la figure 2.

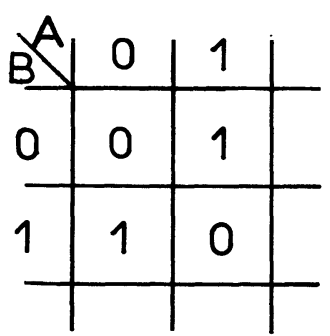

FIG. 2. - Table de vérité de la fonction «ou exclusif».

On voit sur cette table de vérité, que la fonction « ou exclusif » délivre un niveau « 1 » lorsqu'il y a anti-identité entre les nombres $A$ et $B$ à comparer.

b) Circuit de comparaison. - Le compteur de périodes est constitué par trois décades de comptage dans le code naturel BCD (1248). Il permet de régler la durée de chaque impulsion, et de chaque retard, de $\theta$ à $999 \theta$ par pas de $\theta$.

Le circuit de comparaison est constitué de 3 comparateurs décimaux (unités, dizaines, centaines). Les résultats de ces comparaisons sont ensuite combinés par l'intermédiaire d'une porte « et ».

Chaque comparateur décimal est constitué de 4 circuits «ou exclusif » combinés entre eux par une porte « et ».

D'autre part, pour rester en logique positive, chaque comparaison élémentaire se fait par antiidentité ; le nombre logique affiché est donc le conjugué du nombre à détecter. Le schéma de l'ensemble du comparateur est donné figure 3 .

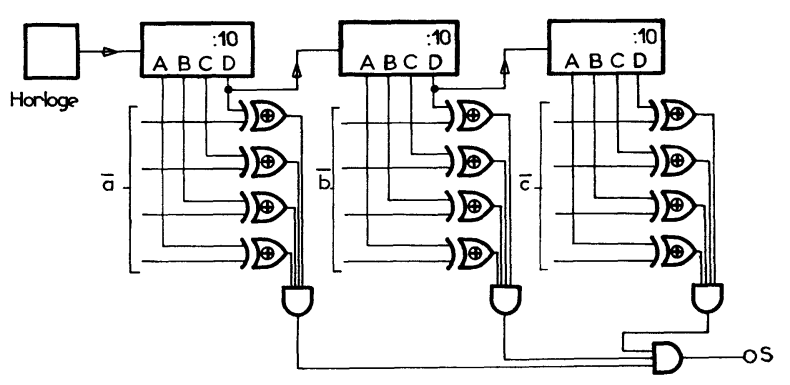

FIG. 3. - Détection par comparaison de la position «cba »du compteur.

3.2 COMmutATion DES VAleuRS AFFiCHÉES. - On remarque sur le schéma représentant le comparateur, que chaque nombre $N$ est représenté sous forme digitale par trois chiffres $\bar{c}, \bar{b}, \bar{a}(\bar{c}=$ centaines, $\bar{b}=$ dizaines, $\bar{a}=$ unités) codés en $\operatorname{BCD}(1,2,4,8)$. Pour passer du nombre $N_{\mathrm{m}}$ au nombre $N_{\mathrm{m}+1}$ suivant il faut opérer la commutation de 12 données logiques.

Le nombre de huit impulsions et de huit écarts. étant fixé, il faut construire un commutateur digital. 
de douze «bits » à seize positions. Ce commutateur est très simple à réaliser à partir de multiplexeurs digitaux 16 «bits» (Fig. 4).

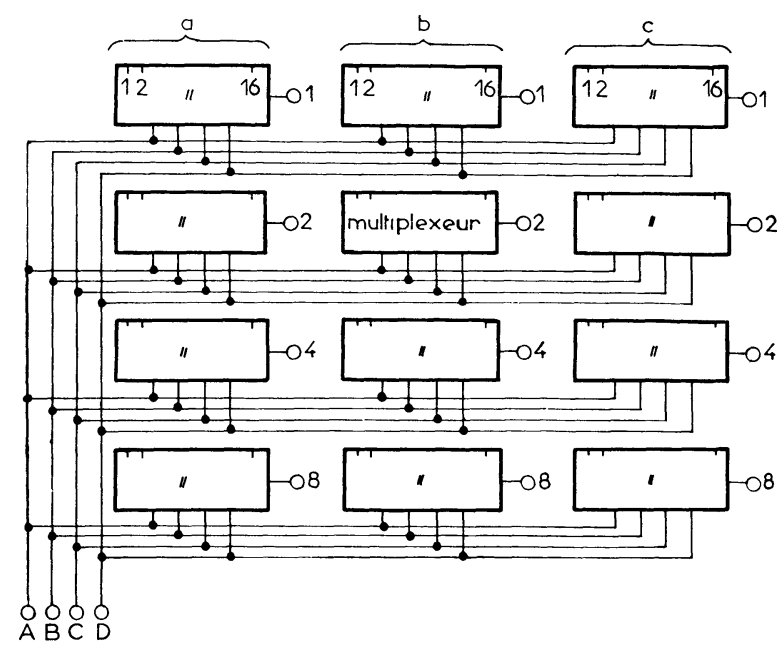

FIG. 4. - Commutateur digital 12 «bits», 16 positions.

Un multiplexeur digital 16 «bits » est un commutateur électronique qui permet d'obtenir en sortie le chiffre binaire appliqué à celle des seize entrées dont l'adresse est ABCD.

Le commutateur d'affichage de la durée de la première impulsion qui permet de programmer sa durée entre 0 et $999 \theta$ comprend trois galettes (unités, dizaines, centaines) à 10 positions codées en $\mathrm{BCD}$ (quatre sorties). Les quatre sorties de la galette des centaines sont reliées aux entrées d'adresse 1 de quatre multiplexeurs délivrant le chiffre des centaines. Les sorties des galettes des dizaines et des unités sont de la même façon reliées aux entrées 1 de huit autres multiplexeurs délivrant par groupe de quatre les chiffres des dizaines et des unités.

Les sorties du commutateur d'affichage de la durée du premier écart sont reliées aux entrées 2 des multiplexeurs et ainsi de suite pour les huit impulsions et les huit écarts.

En modifiant l'adresse ABCD de ces multiplexeurs il est donc possible de commuter électroniquement les différents commutateurs d'affichage. Ce résultat est obtenu à l'aide d'un compteur binaire à quatre « bits » (générateur d'adresse) commandé par l'impulsion de remise à zéro à la fin de chaque cycle de comptage.

3.3 CiRCUIT DE REMiSE A ZÉRo DU COMPTEUR. Pour ne pas introduire d'erreur à chaque fin de cycle de comptage il faut que le compteur soit remis rapidement au zéro dans un temps nettement inférieur à la durée d'une période d'horloge.

Lorsque le nombre de périodes comptées est égal au nombre affiché $N$ le comparateur délivre un niveau logique « 1 » qui remet le compteur à zéro. Ce compteur est constitué d'un ensemble de 12 bascules dont les caractéristiques en régime de commutation ne sont pas identiques. En conséquence dès que l'une de ces bascules est remise à zéro sans que les autres le soient le nombre apparaissant à la sortie du compteur n'est plus $N$ et le comparateur délivre à nouveau un niveau logique « 0 ». La remise à zéro de toutes les bascules n'est pas effectuée.

Pour pallier cet inconvénient on peut maintenir artificiellement l'impulsion de remise à zéro pendant un temps nettement supérieur au temps de commutation de toutes les bascules mais cette solution introduit un retard indésirable. On a préféré détecter l'état zéro de toutes les bascules du compteur et maintenir l'impulsion de remise à zéro tant que les bascules ne sont pas toutes dans cet état. Ce résultat est obtenu à l'aide d'une bascule «Set-Reset» commandée par la sortie du comparateur et par la sortie du circuit indiquant que toutes les bascules sont remises à zéro (Fig. 5). Le fonctionnement du circuit est le suivant :

- Lorsque le compteur a compté un nombre d'impulsions égal au nombre affiché $N$ le comparateur passe en position « 1 » et la sortie $\mathrm{Q}$ de la bascule commandée par l'entrée « Set » passe en position « 1 ». Le niveau est appliqué à la remise à zéro des bascules du compteur. Lorsque toutes les bascules sont remises à zéro un niveau « 1 » arrive sur l'entrée "Reset » et la sortie $Q$ de la bascule passe en position « 0 ». Le comptage peut recommencer.

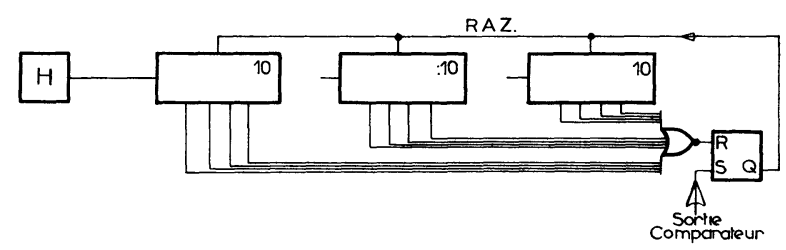

FIG. 5. - Remise à zéro du compteur de cycle.

3.4 COMmutation DeS HORLOGES. - Dans les expériences de R.M.N. pulsée la durée des écarts est en général nettement supérieure à la durée des impulsions et la précision exigée pour cette durée est peu importante.

C'est la raison pour laquelle on a introduit deux horloges internes, une rapide de période $\theta_{i}$ (horloge pilote) pour la durée des impulsions, une dix fois plus lente, de période $\theta_{\mathrm{e}}$, pour la durée des écarts.

Le générateur est prévu pour fonctionner éventuellement avec deux horloges externes. Le circuit de commutation d'horloge est représenté sur la figure 6 .

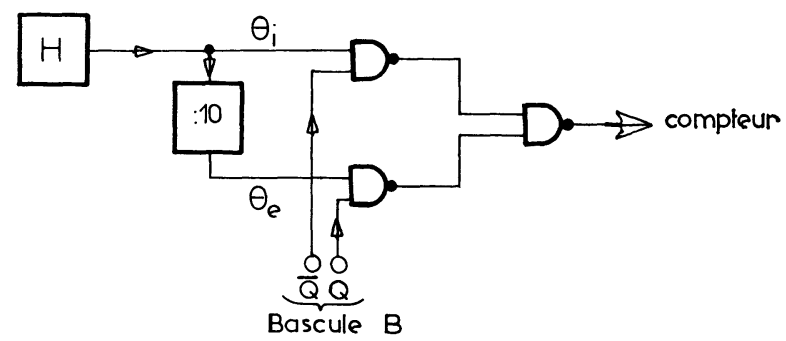

Fig. 6. - Commutation d'horloge $\left(\theta_{\mathrm{e}} \rightarrow\right.$ impulsion ; $\theta_{\mathrm{i}} \rightarrow$ écart $)$. 
La commutation est commandée par la bascule B qui suit le comparateur (Fig. 1).

En position «impulsion » $Q=0, \bar{Q}=1$ le compteur voit l'horloge rapide de période $\theta_{\mathrm{i}}$. En position «Ecart» $Q=1, \bar{Q}=0$ le compteur voit l'horloge de période $\theta_{\mathrm{e}}=10 \theta_{\mathrm{i}}$ ou l'horloge externe.

Le diviseur par 10 est remis à zéro par l'impulsion de remise à zéro des bascules de comptage. On ass_re ainsi la synchronisation de la durée de l'écart sur l'horloge rapide.

3.5 GÉNÉRATEUR DE SÉQUENCE. - Le cycle des huit impulsions et des huit écarts peut être obtenu en fonctionnement relaxé ou déclenché. Dans le premier cas la fin du huitième écart commande le début de la première impulsion. Dans le deuxième cas une impulsion déclenche le démarrage du cycle.

En fonctionnement déclenché il faut réaliser les trois opérations suivantes :

- Interdiction du fonctionnement relaxé.

- Etablissement des conditions initiales. pilote.

- Synchronisation du départ du cycle sur l'horloge

a) Circuit d'interdiction du fonctionnement relaxé. - Le fonctionnement de ce circuit représenté sur la figure 7 est simple. A l'aide de la porte $F$ on détecte le dernier état (soit huitième écart) du compteur binaire « 4 bits » qui génère l'adresse des multiplexeurs et on bloque l'impulsion de sortie du comparateur. Cette impulsion doit normalement provoquer l'avance du générateur d'adresse qui reste maintenant bloqué dans sa dernière position. Il faudra remettre à zéro le compteur binaire « 4 bits » pour que le cycle puisse recommencer.

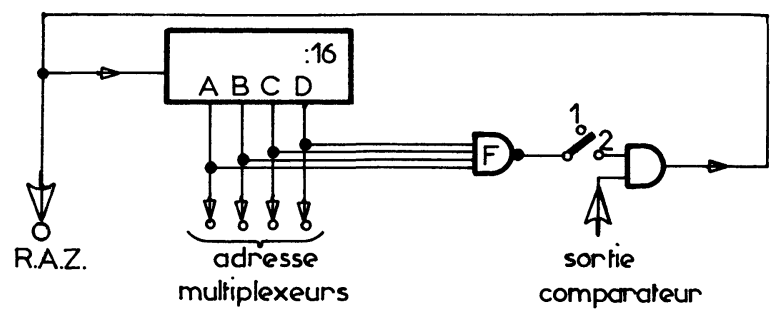

Fig. 7. - Fonctionnement en mode : 1) relaxé ; 2) déclenché.

b) Etablissement des conditions initiales. - L'impulsion $T_{\mathrm{d}}$ de déclenchement du cycle doit déclencher les opérations suivantes :

- Remise à zéro du compteur de périodes.

- Remise à zéro du générateur d'adresse.

- Remise à l'état « 0 » de la bascule $\mathrm{B}$ de sortie (position impulsion).

c) Synchronisation du départ du cycle sur l'horloge. - La récurrence des cycles d'impulsions est synchronisée sur la fréquence du secteur pour éviter des phénomènes de battements.
Le dispositif qui fournit l'impulsion de déclenchement $T_{\mathrm{d}}$ est un compteur qui divise par un nombre variable $M$ une fréquence de $100 \mathrm{~Hz}$ obtenue par multiplication de la fréquence du secteur.

La largeur de l'impulsion de déclenchement est de $10 \mathrm{~ms}$.

Si l'on examine en détail le fonctionnement du générateur d'impulsion à l'instant du déclenchement du cycle on voit que lorsque l'impulsion $T_{\mathrm{d}}$ est appliquée (niveau logique « $1 »)$, le compteur de périodes et le compteur-générateur d'adresse sont remis à zéro mais sont dans l'impossibilité de compter et que la bascule B est dans l'état $Q=0$. A la fin de l'impulsion $T_{\mathrm{d}}$ (niveau logique « $0 »$ ), les compteurs commencent à totaliser les impulsions d'horloge. La durée de la première impulsion est donc de $T_{\mathrm{d}}+N_{1} \theta$. $\left(N_{1}\right.$ nombre de périodes $\theta$ affichées pour la durée de la première impulsion) il faut donc retrancher $T_{\mathrm{d}}$ pour avoir la valeur vraie de la durée. En réalité du fait de la non-synchronisation de l'horloge pilote et du secteur, la durée de la première impulsion n'est définie qu'à une période d'horloge près. Cet effet se comprend aisément à partir des diagrammes représentés figure 8 . Le compteur de périodes passe en
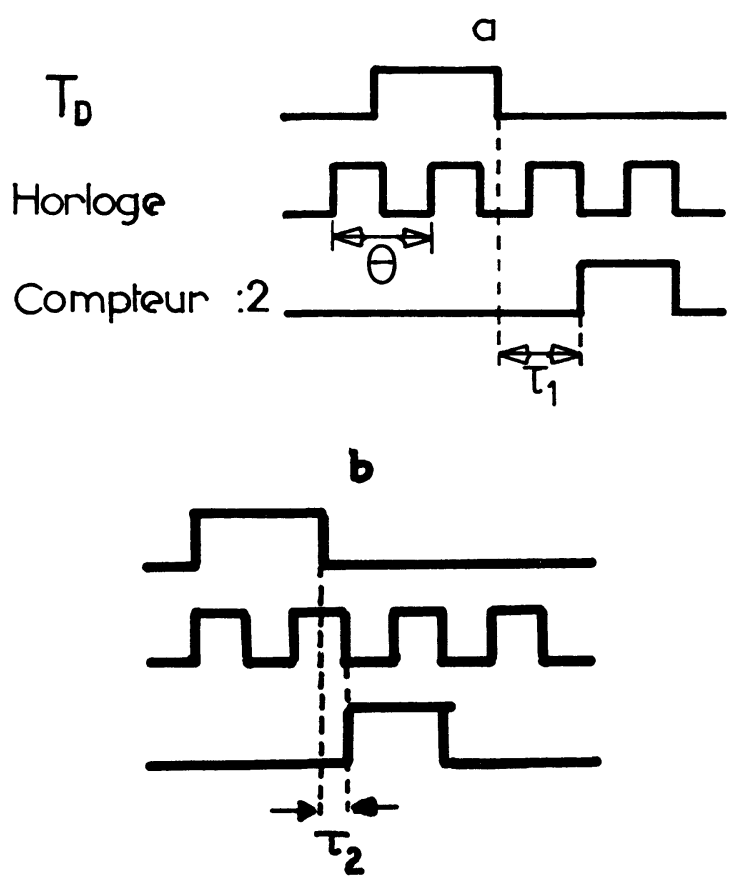

FiG. 8. - Phénomène de « Jitter ».

position « 1 » après un délai qui est suivant le cas $\tau_{1}$ ou $\tau_{2}$ avec $0<\tau_{1}<\theta$ et $0<\tau_{2}<\theta$. Pour éviter ce phénomène de " litter» on a réalisé un circuit de synchronisation qui prolonge l'impulsion $T_{\mathrm{d}}$ jusqu'à la fin de l'impulsion d'horloge qui suit la fin de l'impulsion $T_{\mathrm{d}}$. Ce circuit est représenté sur la figure 9 et le principe de fonctionnement est le suivant :

- Une bascule « Maître-Esclave » est attaquée de manière synchrone par l'horloge pilote et de manière asynchrone par le générateur des impulsions $T_{\mathrm{d}}$. 
Lorsqu'il y a une impulsion $T_{\mathrm{d}}\left(\bar{T}_{\mathrm{d}}\right.$ au niveau logique « 0 »), la sortie $\overline{\mathrm{Q}}_{2}$ de la bascule passe à l'état « 1 » quel que soit le signal d'entrée synchrone (priorité au mode asynchrone). Quand il n'y a plus d'impulsion $T_{\mathrm{d}}\left(\bar{T}_{\mathrm{d}}\right.$ au niveau logique $\left.« 1 »\right)$, la sortie $\overline{\mathrm{Q}}_{2}$ reste dans l'état « 1 » jusqu'à la fin de l'impulsion d'horloge suivante. La bascule Maître-Esclave délivre donc une impulsion $T_{\mathrm{d}}^{\prime}$ de longueur variable dont la fin correspond toujours à la fin d'une impulsion d'horloge pilote (Fig. 10). Pour obtenir la vraie valeur $\mathrm{N} \theta$ de la première impulsion il suffit de bloquer la sortie du générateur d'impulsions pendant le temps $T_{\mathrm{d}}$ ce résultat est obtenu simplement à l'aide d'une porte « ET» (Fig. 11).

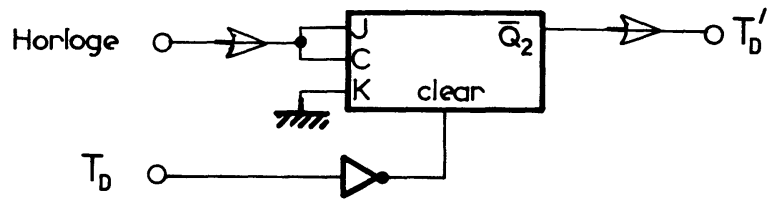

Fig. 9. - Circuit de synchronisation.

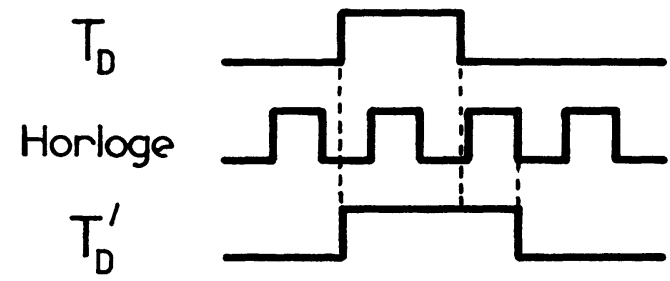

FIG. 10. - Allure de $T_{\mathrm{D}}^{\prime}$ en fonction de $T_{\mathrm{D}}$.

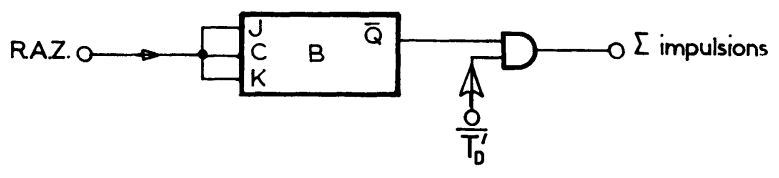

Fig. 11. - Circuit de sortie du générateur.

3.6 CirCuITS DE SORTIE PARALlèLE DES IMPULSIONS - Le générateur d'impulsions tel qu'il est conçu fournit huit impulsions de longueur variable de manière séquentielle. Dans de nombreuses expériences, autres que la R. M. N. pulsée, il est intéressant de pouvoir disposer séparément de chaque impulsion ou d'une combinaison d'un certain nombre d'impulsions (sortie parallèle). On a donc complété le générateur par un circuit qui permet d'obtenir à la sortie une impulsion, ou un groupe quelconque d'impulsions. L'isolement des impulsions est obtenu simplement en utilisant le générateur d'adresse; on a vu en effet qu'à chaque impulsion et à chaque écart correspond un état de ce générateur (voir la table de vérité Fig. 12). Un décodeur décimal relié au diviseur par 16 de la façon représentée à la figure 13 détecte chaque état pair et permet d'isoler chaque impulsion. (Il faut comme précédemment retrancher $T_{\mathrm{d}}^{\prime}$ de la durée de la première impulsion.)

\begin{tabular}{l|l|l|l|l|} 
& $A$ & $B$ & $C$ & $D$ \\
\hline$I_{1}$ & 0 & 0 & 0 & 0 \\
\hline$E_{1}$ & 1 & 0 & 0 & 0 \\
\hline$I_{2}$ & 0 & 1 & 0 & 0 \\
\hline$E_{2}$ & 1 & 1 & 0 & 0 \\
\hline$I_{3}$ & 0 & 0 & 1 & 0 \\
\hline$E_{3}$ & 1 & 0 & 1 & 0 \\
\hline$I_{4}$ & 0 & 1 & 1 & 0 \\
\hline$E_{4}$ & 1 & 1 & 1 & 0 \\
\hline$I_{5}$ & 0 & 0 & 0 & 1 \\
\hline$E_{5}$ & 1 & 0 & 0 & 1 \\
\hline$I_{6}$ & 0 & 1 & 0 & 1 \\
\hline$E_{6}$ & 1 & 1 & 0 & 1 \\
\hline$I_{7}$ & 0 & 0 & 1 & 1 \\
\hline$E_{7}$ & 1 & 0 & 1 & 1 \\
\hline$I_{8}$ & 0 & 1 & 1 & 1 \\
\hline$E_{8}$ & 1 & 1 & 1 & 1 \\
\hline
\end{tabular}

FIG. 12. - Table de vérité du : 16.

Huit interrupteurs permettent à l'aide d'une porte NAND à huit entrées d'obtenir une combinaison d'impulsions (Fig. 13).

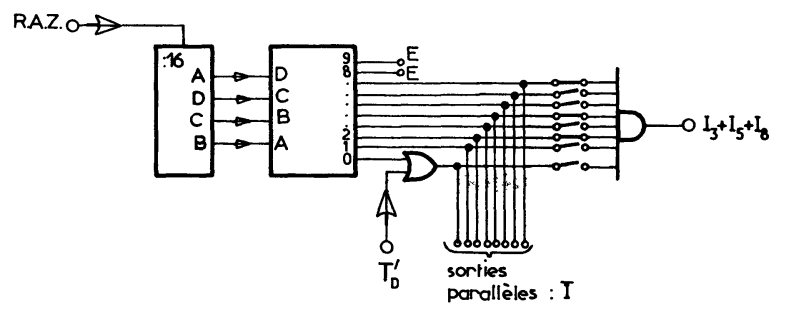

FIG. 13. - Sorties parallèles et combinaison d'impulsions.

4. Performances de l'appareil. - L'appareil fonctionne avec une horloge pilote interne de $10 \mathrm{MHz}$ (ou avec une horloge externe de fréquence $f<10 \mathrm{MHz}$ ). Il permet de générer 8 impulsions de durée réglable entre $0,1 \mu \mathrm{s}$ et $99,9 \mu \mathrm{s}$ par pas de $0,1 \mu \mathrm{s}$. Chaque écart entre impulsions est réglable entre $1 \mu$ s et $999 \mu$ s par pas de $1 \mu$ s (avec possibilité de branchement d'un générateur externe de fréquence quelconque inférieure à $10 \mathrm{MHz}$ ). La sortie séquentielle des impulsions peut être obtenue en mode relaxé ou en mode déclenché avec une période variable de $10 \mathrm{~ms}$ à $99 \mathrm{~s}$ par pas de $10 \mathrm{~ms}$.

La plupart des circuits logiques utilisés sont du type TTL rapides. Les compteurs de périodes sont du type synchrone pour éviter au décodage des impulsions parasites dues aux délais différents des bascules. 


\section{Bibliographie}

[1] Hahn (E. L.), Phys. Rev., 1950, 80, 580.

[2] Carr (H. Y.) and Purcell (E. M.), Phys. Rev., 1954, 94, 630 .

[3] Waugh (J. S.), Wang (C. H.), Huber (L. M.) et Vold (R. L.), J. of Chem. Phys., 1968, 48, 662.
[4] Lalanne (P.) and Eletr (S.), Rev. of Sci. Inst., 1970, 41, 71.

Franconi and Terenzi (M.), A programmed digital IC pulse generator for transient N. M. R. spectrometry.

Rev. of Sci. Inst., 1970, 41, 456. 